\title{
Separability criteria based on the realignment of density matrices and reduced density matrices
}

\author{
Shu-Qian Shen ${ }^{1 *}$, Meng-Yuan Wang ${ }^{1}$, Ming Li $^{1}$, Shao-Ming Fei ${ }^{2,3}$ \\ ${ }^{1}$ College of Science, China University of Petroleum, 266580 Qingdao, P.R. China \\ ${ }^{2}$ School of Mathematical Sciences, Capital Normal University, 100048 Beijing, P.R. China \\ ${ }^{3}$ Max-Planck-Institute for Mathematics in the Sciences, 04103 Leipzig, Germany
}

\begin{abstract}
By combining a parameterized Hermitian matrix, the realignment matrix of the bipartite density matrix $\rho$ and the vectorization of its reduced density matrices, we present a family of separability criteria, which are stronger than the computable cross norm or realignment (CCNR) criterion. With linear contraction methods, the proposed criteria can be used to detect the multipartite entangled states that are biseparable under any bipartite partitions. Moreover, we show by examples that the presented multipartite separability criteria can be more efficient than the corresponding multipartite realignment criterion based on CCNR, multipartite correlation tensor criterion and multipartite covariance matrix criterion.
\end{abstract}

\section{Introduction}

Quantum entanglement, as an intrinsical feature of quantum mechanics, provides the basic physical resource in quantum information and computation [1. It leads to a fundamental problem of how to distinguish between entangled states and separable states. Nevertheless this problem is extremely difficult to solve and has been proven to be NP-hard [2]. In the last decades, a variety of operational methods have been proposed to detect entanglement such as the positive partial transpose (PPT) criterion or PeresHorodecki criterion [3, 4, realignment criteria [5, 6, 7], correlation matrix or tensor criteria [8, 9, 10, 11], covariance matrix criteria [12, 13], entanglement witnesses [4, 14, separability criteria via measurements [15] and so on; see, e.g., [16] for comprehensive surveys.

Among the criteria mentioned above, the most popular one is the PPT criterion [3], which bases on the fact that the partial transpose of a separable state is positive semidefinite. Moreover, this criterion is sufficient and necessary for the separability of $m \times n$ quantum sates with $m n \leq 6$ [4]. However, it only provides necessary conditions for separability of states with higher dimensions, since there exist entangled $2 \times 4$ and $3 \times 3$ states with positive partial transposes [17]. Thus, it is crucial to check whether a given PPT state is entangled or not with $m n>6$.

${ }^{*}$ E-mail: sqshen@upc.edu.cn. 
Another well-known one is the computable cross norm or realignment (CCNR) criterion [5, 6], which is very easy to apply and shows a dramatic ability to detect many PPT entangled states. The multipartite case of this criterion was considered in [18. The authors showed that the partial realignment can detect the tripartite entangled states with biseparability under any bipartite partitions, and that the PPT criterion and the CCNR criterion are equivalent to the permutations of the density matrix's indices. After that, the generalizations of CCNR criterion were investigated in [19]. In [20], the authors made use of the symmetric function of Schmidt coefficients to improve the CCNR criterion further. Recently, the CCNR criterion was used to study the entanglement conditions for any two-mode continuous-variable state with permutational symmetry [21].

In [7, Zhang et al. presented a separability criterion (for simplicity, we call it the Z-R criterion) based on the entry realignment of $\rho-\rho^{A} \otimes \rho^{B}$, which was shown to be strictly stronger than the CCNR criterion and the correlation matrix criterion [8]. A generalization of Z-R criterion was studied in [22]. However, the Z-R criterion is still a strong one.

In this paper, based on a parameterized Hermitian matrix, the realignment matrix of the bipartite density matrix $\rho$ and the vectorization of reduced density matrices $\rho^{A}$ and

$\rho^{B}$, we construct realignment matrices with larger scales. Then, separability criteria for bipartite quantum systems that are stronger than the CCNR criterion are presented. Meanwhile, the new criteria exhibit comparative detection abilities of entanglement compared with the Z-R criterion. Finally, by linear contraction methods introduced in [18, the proposed criteria are valid to detect the multipartite entanglement states that are biseparable under any bipartite partitions, while the Z-R criterion fails to be applied in a similar way. Moreover, two examples show that the obtained multipartite separability criteria can outperform the corresponding multipartite realignment criterion based on CCNR [18, multipartite correlation tensor criterion [10, 11], and multipartite covariance matrix criterion [13].

The remainder of the paper is arranged as follows. In Section 2, we first give the realignment methods, and then introduce the new separability criteria. Theoretical analysis and an example are employed to show the efficiency of the presented criteria. In Section 3, the proposed criteria in Section 2 are extended to the multipartite case. Meanwhile, two examples are supplemented to show the performance of the multipartite separability criteria. Finally, some concluding remarks are made in Section 4.

\section{Separability criteria for bipartite states}

For a matrix $X=\left(x_{i j}\right) \in \mathbb{C}^{m \times n}$, the vector $\operatorname{vec}(X)$ is defined as

$$
\operatorname{vec}(X)=\left(x_{11}, \cdots, x_{m 1}, x_{12}, \cdots, x_{m 2}, \cdots, x_{1 n}, \cdots, x_{m n}\right)^{T},
$$

where $T$ stands for the transpose. Let $Y$ be an $m \times m$ block matrix with $n \times n$ subblocks $Y_{i, j}, i, j=1, \cdots, m$. Then the realignment matrix of $Y[6]$ is defined as

$$
\mathcal{R}(Y)=\left(\operatorname{vec}\left(Y_{1,1}\right) \cdots, \operatorname{vec}\left(Y_{m, 1}\right), \cdots, \operatorname{vec}\left(Y_{1, m}\right), \cdots, \operatorname{vec}\left(Y_{m, m}\right)\right)^{T} .
$$


For any quantum state $\rho$ in $\mathbb{C}^{d_{A}} \otimes \mathbb{C}^{d_{B}}$, we define

$$
\mathcal{N}_{\alpha, \ell}^{G}(\rho)=\left(\begin{array}{cc}
G & \alpha \omega_{\ell}\left(\rho^{B}\right)^{T} \\
\alpha \omega_{\ell}\left(\rho^{A}\right) & \mathcal{R}(\rho)
\end{array}\right)
$$

where $G$ is a given Hermitian matrix, $\alpha$ is an arbitrary real number, $\ell$ is an arbitrary natural number, $\rho^{A}\left(\rho^{B}\right)$ is the reduced density matrix of the $A(B)$ subsystem, and for any complex matrix $X$,

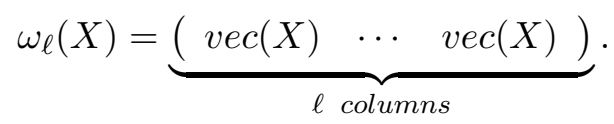

We denote by $\|\cdot\|_{\mathrm{tr}},\|\cdot\|_{2}, \operatorname{Tr}(\cdot)$ and $E_{m \times n}$ the trace norm (i.e. the sum of singular values), the spectral norm (i.e. the maximum singular value), the trace and the $m \times n$ matrix with all entries being 1 , respectively. The following theorem establishes the new separability criterion based on $\mathcal{N}_{\alpha, \ell}^{G}(\rho)$ for bipartite states.

Theorem 2.1. Let $G-\alpha^{2} E_{\ell \times \ell}$ be positive semidefinite. If the state $\rho$ in $\mathbb{C}^{d_{A}} \otimes \mathbb{C}^{d_{B}}$ is separable, then

$$
\left\|\mathcal{N}_{\alpha, \ell}^{G}(\rho)\right\|_{\operatorname{tr}} \leq 1+\operatorname{Tr}(G) .
$$

Proof. Since $\rho$ is separable, it can be written as a convex combination of pure product states, i.e.,

$$
\rho=\sum_{i} p_{i} \rho_{i}^{A} \otimes \rho_{i}^{B}
$$

where $0 \leq p_{i} \leq 1, \sum_{i} p_{i}=1, \rho_{i}^{A}$ and $\rho_{i}^{B}$ are pure states of the $A$ and $B$ subsystems, respectively. One gets

$$
\left\|\mathcal{N}_{\alpha, \ell}^{G}(\rho)\right\|_{\mathrm{tr}}=\left\|\sum_{i} p_{i} \mathcal{N}_{\alpha, \ell}^{G}\left(\rho_{i}^{A} \otimes \rho_{i}^{B}\right)\right\|_{\mathrm{tr}} \leq \sum_{i} p_{i}\left\|\mathcal{N}_{\alpha, \ell}^{G}\left(\rho_{i}^{A} \otimes \rho_{i}^{B}\right)\right\|_{\mathrm{tr}} .
$$

Thus, we only need to give the upper bound of

$$
\left\|\mathcal{N}_{\alpha, \ell}^{G}\left(\rho_{i}^{A} \otimes \rho_{i}^{B}\right)\right\|_{\operatorname{tr}}=\left\|\left(\begin{array}{cc}
G & \alpha \omega_{\ell}\left(\rho_{i}^{B}\right)^{T} \\
\alpha \omega_{\ell}\left(\rho_{i}^{A}\right) & \operatorname{vec}\left(\rho_{i}^{A}\right) \operatorname{vec}\left(\rho_{i}^{B}\right)^{T}
\end{array}\right)\right\|_{\mathrm{tr}},
$$

where we have used the equality [6]

$$
\mathcal{R}\left(\rho_{i}^{A} \otimes \rho_{i}^{B}\right)=\operatorname{vec}\left(\rho_{i}^{A}\right) \operatorname{vec}\left(\rho_{i}^{B}\right)^{T} .
$$

Since the equality $\left.\|\operatorname{vec}(|x\rangle\langle x|)\|_{2}=\|\| x\right\rangle \|_{2}^{2}$ holds for any vector $|x\rangle$, there exist unitary matrices $U$ and $V$ such that

$$
\begin{aligned}
& U \operatorname{vec}\left(\rho_{i}^{A}\right)=\left(\begin{array}{llll}
\left\|\operatorname{vec}\left(\rho_{i}^{A}\right)\right\|_{2} & 0 & \cdots & 0
\end{array}\right)^{T}=\left(\begin{array}{lllll}
1 & 0 & \cdots & 0
\end{array}\right)^{T}, \\
& V \operatorname{vec}\left(\rho_{i}^{B}\right)=\left(\begin{array}{lllll}
\left\|\operatorname{vec}\left(\rho_{i}^{B}\right)\right\|_{2} & 0 & \cdots & 0
\end{array}\right)^{T}=\left(\begin{array}{llll}
1 & 0 & \cdots & 0
\end{array}\right)^{T} .
\end{aligned}
$$


Furthermore, we have

$$
\left(\begin{array}{cc}
I_{\ell} & \\
& U
\end{array}\right) \mathcal{N}_{\alpha, \ell}^{G}\left(\rho_{i}^{A} \otimes \rho_{i}^{B}\right)\left(\begin{array}{cc}
I_{\ell} & \\
& V^{\dagger}
\end{array}\right)=\left(\begin{array}{cc}
G & \alpha N \\
\alpha M & P
\end{array}\right):=S_{\alpha, \ell}^{G},
$$

where $I_{\ell}$ is the $\ell \times \ell$ identity matrix, and

$$
M=\left(\begin{array}{cccc}
1 & 1 & \cdots & 1 \\
0 & 0 & \cdots & 0 \\
\vdots & \vdots & \ddots & \vdots \\
0 & 0 & \cdots & 0
\end{array}\right)_{d_{A}^{2} \times \ell}, N=\left(\begin{array}{cccc}
1 & 0 & \cdots & 0 \\
1 & 0 & \cdots & 0 \\
\vdots & \vdots & \ddots & \vdots \\
1 & 0 & \cdots & 0
\end{array}\right)_{\ell \times d_{B}^{2}}, P=\left(\begin{array}{cccc}
1 & 0 & \cdots & 0 \\
0 & 0 & \cdots & 0 \\
\vdots & \vdots & \ddots & \vdots \\
0 & 0 & \cdots & 0
\end{array}\right)_{d_{A}^{2} \times d_{B}^{2}} .
$$

The matrix $S_{\alpha, \ell}^{G}$ can be repartitioned as

$$
S_{\alpha, \ell}^{G}=\left(\begin{array}{cc}
\mathcal{W}_{\alpha, \ell}^{G} & 0 \\
0 & 0
\end{array}\right)
$$

where

$$
\mathcal{W}_{\alpha, \ell}^{G}=\left(\begin{array}{cc}
G & \alpha E_{\ell \times 1} \\
\alpha E_{1 \times \ell} & 1
\end{array}\right)
$$

Since the matrix $G-\alpha^{2} E_{\ell \times \ell}$ is positive semidefinite, from [23, Theorem 7.7.6], $\mathcal{W}_{\alpha, \ell}^{G}$ is also positive semidefinite. Due to the fact that the trace norm of a Hermitian positive semidefinite matrix equals to its trace, we get, by (2) and (3),

$$
\left\|\mathcal{N}_{\alpha, \ell}^{G}\left(\rho_{i}^{A} \otimes \rho_{i}^{B}\right)\right\|_{\mathrm{tr}}=\left\|S_{\alpha, \ell}^{G}\right\|_{\mathrm{tr}}=\left\|\mathcal{W}_{\alpha, \ell}^{G}\right\|_{\mathrm{tr}}=1+\operatorname{Tr}(G),
$$

and then, by (1),

$$
\left\|\mathcal{N}_{\alpha, \ell}^{G}(\rho)\right\|_{\operatorname{tr}} \leq 1+\operatorname{Tr}(G)
$$

The CCNR criterion [5, 6] claims that any separable state $\rho$ in $\mathbb{C}^{d_{A}} \otimes \mathbb{C}^{d_{B}}$ satisfies the inequality

$$
\|\mathcal{R}(\rho)\|_{\text {tr }} \leq 1 .
$$

It is obvious that Theorem 2.1 reduces to the CCNR criterion when $\alpha$ is chosen to be 0 . For the case $\alpha \neq 0$, the following proposition shows that Theorem 2.1 is stronger than the CCNR criterion.

Proposition 2.1. Theorem 2.1 is stronger than the CCNR criterion when $\alpha \neq 0$.

Proof. For any state $\rho$ in $\mathbb{C}^{d_{A}} \otimes \mathbb{C}^{d_{B}}$, we have, by [8, Lemma 1],

$$
\left\|\mathcal{N}_{\alpha, \ell}^{G}(\rho)\right\|_{\text {tr }} \geq\|G\|_{\text {tr }}+\|\mathcal{R}(\rho)\|_{\text {tr }}=\operatorname{Tr}(G)+\|\mathcal{R}(\rho)\|_{\text {tr }} .
$$

Thus, if $\left\|\mathcal{N}_{\alpha, \ell}^{G}(\rho)\right\|_{\text {tr }} \leq 1+\operatorname{Tr}(G)$, one has $\|\mathcal{R}(\rho)\|_{\text {tr }} \leq 1$.

By choosing some special parameterized matrices $G$, we obtain the following two corollaries for detecting entanglement of bipartite states. 
Corollary 2.1. If the state $\rho$ in $\mathbb{C}^{d_{A}} \otimes \mathbb{C}^{d_{B}}$ is separable, then

$$
\left\|\mathcal{N}_{\alpha, \ell}^{\ell \alpha^{2} I_{\ell}}(\rho)\right\|_{\operatorname{tr}} \leq 1+\ell^{2} \alpha^{2}
$$

Proof. We take $G=\ell \alpha^{2} I_{\ell}$. Obviously $G-\alpha^{2} E_{\ell \times \ell}$ is positive semidefinite. Then, from Theorem 2.1, the conclusion holds.

Corollary 2.2. If the state $\rho$ in $\mathbb{C}^{d_{A}} \otimes \mathbb{C}^{d_{B}}$ is separable, then

$$
\left\|\mathcal{N}_{\alpha, \ell}^{\alpha^{2} E_{\ell \times \ell}}(\rho)\right\|_{\operatorname{tr}} \leq 1+\ell \alpha^{2}
$$

Proof. From $G=\alpha^{2} E_{\ell \times \ell}$, we get $G-\alpha^{2} E_{\ell \times \ell}=0$. Hence the conclusion holds by Theorem 2.1.

In the following, we consider the problem of the selection of $\ell$. By adding a row and a column to $G$, we get an $(\ell+1) \times(\ell+1)$ Hermitian matrix

$$
\bar{G}=\left(\begin{array}{cc}
\tau & \eta^{\dagger} \\
\eta & G
\end{array}\right)
$$

where $\eta \in \mathbb{C}^{\ell}, \tau \in \mathbb{R}$. By an analogous proof of Proposition 2.1, we can derive a more general result immediately.

Proposition 2.2. If $\bar{G}-\alpha^{2} E_{(\ell+1) \times(\ell+1)}$ is positive semidefinite, then the separability criterion $\left\|\mathcal{N}_{\alpha, \ell+1}^{\bar{G}}(\rho)\right\|_{\operatorname{tr}} \leq 1+\operatorname{Tr}(\bar{G})$ is stronger than the separability criterion $\left\|\mathcal{N}_{\alpha, \ell}^{G}(\rho)\right\|_{\mathrm{tr}} \leq 1+\operatorname{Tr}(G)$.

From Proposition 2.2, it is obvious that Corollaries 2.1-2.2 can detect more entanglement when $\ell$ gets larger.

The Z-R criterion given in [7] is based on the realignment of $\rho-\rho^{A} \otimes \rho^{B}$. It states that, for any separable state $\rho$ in $\mathbb{C}^{d_{A}} \otimes \mathbb{C}^{d_{B}}$, the inequality

$$
\left\|\mathcal{R}\left(\rho-\rho^{A} \otimes \rho^{B}\right)\right\|_{\operatorname{tr}} \leq \sqrt{\left(1-\operatorname{Tr}\left(\left(\rho^{A}\right)^{2}\right)\right)\left(1-\operatorname{Tr}\left(\left(\rho^{B}\right)^{2}\right)\right)}
$$

must hold. This criterion is also stronger than the CCNR criterion, but the exact relation between this criterion and Theorem 2.1 needs to be established.

The following well-known example shows the power of Corollaries 2.1 and 2.2. Nevertheless, we only report the results from Corollary 2.1, since Corollary 2.1 has a close performance to Corollary 2.2 by numerical calculations.

Example 2.1. The following $3 \times 3$ PPT entangled state was introduced in [24]:

$$
\rho=\frac{1}{4}\left(I_{9}-\sum_{k=0}^{4}\left|\xi_{k}\right\rangle\left\langle\xi_{k}\right|\right),
$$


where

$$
\begin{aligned}
& \left|\xi_{0}\right\rangle=\frac{1}{\sqrt{2}}|0\rangle(|0\rangle-|1\rangle),\left|\xi_{1}\right\rangle=\frac{1}{\sqrt{2}}(|0\rangle-|1\rangle)|2\rangle,\left|\xi_{2}\right\rangle=\frac{1}{\sqrt{2}}|2\rangle(|1\rangle-|2\rangle), \\
& \left|\xi_{3}\right\rangle=\frac{1}{\sqrt{2}}(|1\rangle-|2\rangle)|0\rangle,\left|\xi_{4}\right\rangle=\frac{1}{3}(|0\rangle+|1\rangle+|2\rangle)(|0\rangle+|1\rangle+|2\rangle) .
\end{aligned}
$$

We consider the mixture of $\rho$ with white noise:

$$
\rho_{p}=\frac{1-p}{9} I_{9}+p \rho
$$

The CCNR criterion and the Z-R criterion can detect entanglement of $\rho_{p}$ for $0.8897 \leq$ $p \leq 1$ and $0.8822 \leq p \leq 1$, respectively. The latter entanglement condition $0.8822 \leq p \leq$ 1 can also be obtained by Corollary 2.1 when one of the conditions $\ell \geq 12, \alpha \geq 3.4640$ and $\ell \geq 1, \alpha \geq 11.6590$ holds.

Although Example 2.1 shows that Corollary 2.1 is not better than the Z-R criterion, their detection abilities for entanglement are comparative. More importantly, Theorem 2.1 can be extended to multipartite states by linear contraction methods [18] directly, see Section 3.

\section{Separability criteria for multipartite states}

In this section, Theorem 2.1 and its corollaries are applied for multipartite systems. An $n$ partite sate $\rho$ in $\mathbb{C}^{d_{1}} \otimes \cdots \otimes \mathbb{C}^{d_{n}}$ is separable (or fully separable) 25] if and only if it can be represented as

$$
\rho=\sum_{i} p_{i} \rho_{i}^{1} \otimes \cdots \otimes \rho_{i}^{n}
$$

where $p_{i} \geq 0, \sum_{i} p_{i}=1$, and $\rho_{i}^{1}, \cdots, \rho_{i}^{n}$ are pure states of subsystems.

Under the condition that the linear map $\mathcal{L}_{(k)}$ acting on the $k$ chosen subsystems is contractive on product states $\sigma_{j_{1}} \otimes \sigma_{j_{2}} \otimes \cdots \otimes \sigma_{j_{k}}$, where $1 \leq j_{1}<j_{2}<\cdots<j_{k} \leq n$, Horodecki et al. [18] presented the following separability criterion: if a state $\rho$ in $\mathbb{C}^{d_{1}} \otimes \cdots \otimes \mathbb{C}^{d_{n}}$ is separable, then

$$
\left\|\mathcal{L}_{(k)} \otimes \mathcal{I}_{(n-k)}(\rho)\right\|_{\text {tr }} \leq 1,
$$

where the map $\mathcal{I}_{(n-k)}$ means that the remaining $n-k$ subsystems are left untouched.

To extend Theorem 2.1 to the multipartite case, we define the following map:

$$
\mathcal{M}_{\alpha, \ell}^{G}(\rho)=\frac{1}{1+\operatorname{Tr}(G)}\left(\begin{array}{cc}
\operatorname{Tr}(\rho) G & \alpha \omega_{\ell}\left(\rho^{B}\right)^{T} \\
\alpha \omega_{\ell}\left(\rho^{A}\right) & \mathcal{R}(\rho)
\end{array}\right), \forall \rho \text { in } \mathbb{C}^{d_{A}} \otimes \mathbb{C}^{d_{B}}
$$

where $G, \ell$ and $\alpha$ are defined as in Theorem 2.1. $\operatorname{Tr}(\rho)$ is only to guarantee the linearity of the map in the extension. From Theorem 2.1, the map (5) is contractive on any product state $\sigma^{A} \otimes \sigma^{B}$. Thus, due to Horodeckis' separability criterion (4) for multipartite systems, we get the following separability criterion based on $\mathcal{M}_{\alpha, \ell}^{G}$. 
Theorem 3.1. If the state $\rho$ in $\mathbb{C}^{d_{1}} \otimes \cdots \otimes \mathbb{C}^{d_{n}}$ is separable, then

$$
\left\|\mathcal{M}_{\alpha, \ell}^{G,(2)} \otimes \mathcal{I}_{(n-2)}(\rho)\right\|_{t r} \leq 1,
$$

where $\mathcal{M}_{\alpha, \ell}^{G,(2)}$ denotes the map $\mathcal{M}_{\alpha, \ell}^{G}$ acting on any chosen 2 subsystems.

Under the combination of the CCNR criterion and (4), Horodecki et al. [18] showed that, if the state $\rho$ in $\mathbb{C}^{d_{1}} \otimes \cdots \otimes \mathbb{C}^{d_{n}}$ is separable, then

$$
\left\|\mathcal{R}^{(2)} \otimes \mathcal{I}_{(n-2)}(\rho)\right\|_{\text {tr }} \leq 1
$$

where $\mathcal{R}^{(2)}$ denotes the realignment map $\mathcal{R}$ acting on any chosen 2 subsystems. For simplicity, we call it the H-R criterion. Surprisingly, this criterion can detect the tripartite entangled state which is biseparable under any bipartite partitions [18]. From Proposition 2.1, it can be found that the H-R criterion should be weaker than Theorem 3.1.

The Z-R criterion is based on the realignment of $\rho-\rho^{A} \otimes \rho^{B}$, but this realignment is not linear on quantum states. Hence, the Z-R criterion cannot be extended to the multipartite case by contraction methods. Nevertheless, the Z-R criterion can be generalized to an analog of permutation separability criterion for multipartite systems [7. However, the obtained multipartite separability criterion is only valid for systems of even number of subsystems.

The following examples illustrate the efficiency of Theorem 3.1 compared with the H-R criterion [18, the multipartite correlation tensor criteria [10, Theorem 1] and [11, Theorem 4], and the multipartite covariance matrix criterion [13, Proposition 2].

Example 3.1. Consider the tripartite state [24]:

$$
\rho_{A B C}=\frac{1}{8}\left(I_{8}-\sum_{k=1}^{4}\left|\phi_{k}\right\rangle\left\langle\phi_{k}\right|\right),
$$

where

$$
\left|\phi_{1}\right\rangle=|0,1,+\rangle,\left|\phi_{2}\right\rangle=|1,+, 0\rangle,\left|\phi_{3}\right\rangle=|+, 0,1\rangle,\left|\phi_{4}\right\rangle=|-,-,-\rangle, \pm=\frac{1}{\sqrt{2}}(|0\rangle \pm|1\rangle) .
$$

It was shown in 24] that this state is biseparable under any bipartite partitions $A \mid B C$, $B \mid C A$ and $C \mid A B$, but it is still entangled.

To verify the efficiency of Theorem 3.1, we consider the mixture of $\rho_{A B C}$ with white noise:

$$
\rho_{A B C}^{p}=\frac{1-p}{8} I_{8}+p \rho_{A B C} .
$$

Clearly, $\rho_{A B C}^{p}$ is also biseparable under any bipartite partitions $A|B C, B| C A$ and $C \mid A B$. 
Let $\mathcal{R}_{(B C)}$ and $\mathcal{M}_{\alpha, \ell}^{G,(B C)}$ be the maps $\mathcal{R}$ and $\mathcal{M}_{\alpha, \ell}^{G}$ acting on $B$ and $C$ subsystems, respectively. By the $\mathrm{H}-\mathrm{R}$ criterion, the entanglement of $\rho_{A B C}^{p}$ for $0.873529 \leq p \leq 1$ can be detected. Meanwhile, from Theorem 3.1 and Corollary 2.1, we choose $G=\ell \alpha^{2} I_{\ell}$. Table 1 displays the entangled conditions for different values of $\alpha$ and $\ell$. It is easy to see that Theorem 3.1 is more efficient than the H-R criterion. Moreover, the detection ability of Theorem 3.1 becomes slightly stronger when $\alpha$ and $\ell$ get larger.

However, the corresponding multipartite correlation tensor criteria [10, 11] and the multipartite covariance matrix criterion [13, Proposition 2] cannot detect any entanglement in $\rho_{A B C}^{p}$.

\begin{tabular}{c|c|c|c|c}
\hline$\alpha$ & $\ell=1$ & $\ell=10$ & $\ell=100$ & $\ell=500$ \\
\hline 1 & $0.845476 \leq p \leq 1$ & $0.831017 \leq p \leq 1$ & $0.828701 \leq p \leq 1$ & $0.828483 \leq p \leq 1$ \\
\hline 10 & $0.828701 \leq p \leq 1$ & $0.828455 \leq p \leq 1$ & $0.828430 \leq p \leq 1$ & $0.828428 \leq p \leq 1$ \\
\hline 100 & $0.828430 \leq p \leq 1$ & $0.828428 \leq p \leq 1$ & $0.828428 \leq p \leq 1$ & $0.828427 \leq p \leq 1$ \\
\hline
\end{tabular}

Table 1: Entanglement conditions of $\rho_{A B C}^{p}$ from Theorem 3.1 with $G=\ell \alpha^{2} I_{\ell}$.

Example 3.2. A perturbation of the GHZ state leads to the following tripartite qubit state used in [13]:

$$
\left|\psi_{G H Z}^{\prime}\right\rangle=\frac{1}{\chi}(|000\rangle+\epsilon|110\rangle+|111\rangle),
$$

where $\epsilon$ is a given real parameter, and $\chi$ denotes the normalization. We consider the mixture of this state with white niose:

$$
\rho_{G H Z^{\prime}}^{p}=\frac{1-p}{8} I_{8}+p\left|\psi_{G H Z}^{\prime}\right\rangle\left\langle\psi_{G H Z}^{\prime}\right| .
$$

In the numerical demonstration, the maps $\mathcal{R}_{(B C)}$ and $\mathcal{M}_{\alpha, \ell}^{G,(B C)}$ are used for the H-R criterion and Theorem 3.1, respectively. From Corollary 2.1, the parameterized matrix $G$ is simply chosen to be $G=\ell \alpha^{2} I_{\ell}$ with $\alpha=\ell=10$. It should be noted that, for tripartite systems, the multipartite correlation tensor criterion [10, Theorem 1] is equivalent to the criterion [11, Theorem 4].

Table 2 gives the results for different values of $\epsilon$. It follows that Theorem 3.1 outperforms the H-R criterion, the multipartite correlation tensor criterion, and the multipartite covariance matrix criterion for different values of $\epsilon$.

\section{Conclusion}

In this paper, by introducing a Hermitian matrix and a real parameter, we first realigned the density matrix and its reduced density matrices, and then proposed a family of separability criteria, which, by a strict proof, are stronger than the well-known CCNR criterion. In general, the new criteria become more efficient when the involved parameter $\ell$ gets larger. Second, due to the special choices of $G$, we gave two simple separability criteria, i.e., Corollaries 2.1 and 2.2, which are easy to apply and exhibit, by examples, comparative abilities of entanglement detection compared with the Z-R criterion. Finally, by linear contraction methods, the presented criteria were extended 


\begin{tabular}{c|c|c|c|c}
\hline$\epsilon$ & H-R & M-T & M-C & Theorem 3.1 \\
\hline 0 & $0.3344 \leq p \leq 1$ & $0.4118 \leq p \leq 1$ & -- & $0.3334 \leq p \leq 1$ \\
\hline $10^{-5}$ & $0.3344 \leq p \leq 1$ & $0.4118 \leq p \leq 1$ & $p=1$ & $0.3334 \leq p \leq 1$ \\
\hline $10^{-3}$ & $0.3344 \leq p \leq 1$ & $0.4118 \leq p \leq 1$ & $0.9981 \leq p \leq 1$ & $0.3334 \leq p \leq 1$ \\
\hline $10^{-1}$ & $0.3340 \leq p \leq 1$ & $0.4118 \leq p \leq 1$ & $0.8341 \leq p \leq 1$ & $0.3339 \leq p \leq 1$ \\
\hline 1 & $0.3899 \leq p \leq 1$ & $0.4256 \leq p \leq 1$ & $0.4286 \leq p \leq 1$ & $0.3849 \leq p \leq 1$ \\
\hline
\end{tabular}

Table 2: Entanglement conditions of $\rho_{G H Z}^{p}$ with different values of $\epsilon$ from the $H$ - $R$ criterion $(H-R)$, the multipartite correlation tensor criterion $(M-T)$, the multipartite covariance matrix criterion $(M-C)$, and Theorem 3.1. The symbol "--" denotes that no entanglement can be detected.

to the multipartite case. Two examples showed that the presented multipartite separability criteria can be more efficient than the H-R criterion, the multipartite correlation tensor criterion, and the multipartite covariance matrix criterion.

There are still many problems that need to be further addressed. For example, the exact relations between Theorem 2.1 and the Z-R criterion should be clarified further. How to choose the parameterized matrix and the parameters $\alpha, \ell$ such that the proposed criteria can detect more entanglement should be further investigated. Whether some other criteria can be generalized and improved by the methods used in this paper is also an interesting problem.

\section{Acknowledgments}

This work is supported by the NSFC (11105226, 11275131), the Fundamental Research Funds for the Central Universities (15CX08011A, 24720122013), and the Projectsponsored by SRF for ROCS, SEM.

\section{References}

[1] M.A. Nielsen and I.L. Chuang, Quantum computation and quantum information, Cambridge University Press, Cambridge, UK, 2010.

[2] L. Gurvits, in Proceedings of the Thirty-Fifth Annual ACM Symposium on Theory of Computing (ACM Press, New York, 2003), pp. 10-19.

[3] A. Peres, Phys. Rev. Lett. 77, 1413 (1996).

[4] M. Horodecki, P. Horodecki, and R. Horodecki, Phys. Lett. A 223, 1 (1996).

[5] O. Rudolph, Phys. Rev. A 67, 032312 (2003); O. Rudolph, Quantum Inf. Process. 4, 219 (2005).

[6] K. Chen and L.A. Wu, Quantum Inf. Comput. 3, 193 (2003).

[7] C.J. Zhang, Y.S. Zhang, S. Zhang, and G.C. Guo, Phys. Rev. A 77, 060301(R) (2008).

[8] J.I. de Vicente, Quantum Inf. Comput. 7, 624 (2007). 
[9] J.I. de Vicente, J. Phys. A: Math. Theor. 41, 065309 (2008); C. Klöckl and M. Huber, Phys. Rev. A 91, 042339 (2015); M. Li, J. Wang, S.M. Fei, and X. Li-Jost, Phys. Rev. A, 89, 022325 (2014).

[10] A.S.M. Hassan and P.S. Joag, Quantum Inf. Comput. 8, 773 (2008).

[11] J.I. de Vicente and M. Huber, Phys. Rev. A 84, 062306 (2011).

[12] O. Gühne, P. Hyllus, O. Gittsovich, and J. Eisert, Phys. Rev. Lett. 99, 130504 (2007); O. Gittsovich, O. Gühne, P. Hyllus, and J. Eisert, Phys. Rev. A 78, 052319 (2008).

[13] O. Gittsovich, P. Hyllus, and O. Gühne, Phys. Rev. A 82, 032306 (2010).

[14] B.M. Terhal, Phys. Lett. A 271, 319 (2000); D. Chruściński and G. Sarbicki, J. Phys. A: Math. Theor. 47, 483001 (2014).

[15] C. Spengler, M. Huber, S. Brierley, T. Adaktylos, and B.C. Hiesmayr, Phys. Rev. A 86, 022311 (2012); B. Chen, T. Ma, and S.M. Fei, Phys. Rev. A 89, 064302 (2014); S.Q. Shen, M. Li, and X.F. Duan, Phys. Rev. A 91, 012326 (2015).

[16] R. Horodecki, P. Horodecki, M. Horodecki, and K. Horodecki, Rev. Mod. Phys. 81, 865 (2009); O. Gühne and G. Tóth, Phys. Rep. 474, 1 (2009).

[17] P. Horodecki, Phys. Lett. A 232, 333 (1997).

[18] M. Horodecki, P. Horodecki, and R. Horodecki, Open Syst. Inf. Dyn. 13, 103 (2006).

[19] K. Chen and L.A. Wu, Phys. Lett. A 306, 14 (2002); S. Albeverio, K. Chen, and S.M. Fei, Phys. Rev. A 68, 062313 (2003).

[20] C. Lupo, P. Aniello, and A. Scardicchio, J. Phys. A: Math. Theor. 41, 415301 (2008); C.K. Li, Y.T. Poon, and N.S. Sze, J. Phys. A: Math. Theor. 44, 315304 (2011).

[21] L.Z. Jiang, X.Y. Chen, P. Yu, and M. Tian, Phys. Rev. A, 89, 012332 (2014).

[22] P. Aniello and C. Lupo, J. Phys. A: Math. Theor. 41, 355303 (2008).

[23] R.A. Horn and C.R. Johnson, Matrix analysis, Cambridge University Press, Cambridge, UK, 1985.

[24] C.H. Bennett, D.P. DiVincenzo, T. Mor, P.W. Shor, J.A. Smolin, and B.M. Terhal, Phys. Rev. Lett. 82, 5385 (1999).

[25] R.F. Werner, Phys. Rev. A 40, 4277 (1989). 